\title{
Melting thin foils by incident relativistic electron bunch
}

\author{
G. Stupakov \\ SLAC National Accelerator Laboratory, Menlo Park, CA 94025
}

June 7, 2013

\section{INTRODUCTION}

We calculate the electric currents induced in a metal foil by an impinging relativistic beam and the foil heating due to the ohmic losses in the foil. Conditions when the foil melts are obtained and calculated for three metals: titanium, beryllium and tungsten, and for several beam parameters relevant for the FACET experiment at SLAC.

We assume that the beam is traveling with the speed of light and hits the foil at right angle.

\section{GREEN'S FUNCTION}

We first consider a unit point charge that hits the wall and find the electric and magnetic fields on the surface. The solution of this problem can be found in $[1]^{1}$. The normal electric and the azimuthal magnetic fields on the surface of the metal for $t>0$ are given by the following equations

$$
E_{z}=-\frac{2}{\sqrt{c^{2} t^{2}-r^{2}}} \delta\left(\sqrt{c^{2} t^{2}-r^{2}}\right), H_{\theta}=\frac{2 c t}{r \sqrt{c^{2} t^{2}-r^{2}}} \delta\left(\sqrt{c^{2} t^{2}-r^{2}}\right),
$$

where we use the cylindrical coordinate system $r, \theta, z$ with the axis $z$ directed from the metal to vacuum, assuming that the charge hits the wall at time

${ }^{1}$ Actually, Chao gives solution of a more general problem of the electromagnetic field excited by a point charge between two parallel metal planes. We are interested in the limit of that solution when the distance between the planes tends to infinity. 
$t=0$ at the origin of the coordinate system $r=0$. Note that the integral

$2 \pi \int_{0}^{\infty} r E_{z} d r=-4 \pi$ which means that the image charge on the metal surface is equal to -1 . From the expression for $H_{\theta}$ we can also find the $x$ and $y$ components of the magnetic field using $H_{x}=-H_{\theta} y / r$ and $H_{y}=H_{\theta} x / r$.

Expressions (1) can now be used as the Green functions for calculation of the magnetic field on the metal surface induced by a relativistic bunch. For this we need to assume that the charge hits the wall at point $x^{\prime}, y^{\prime}$ at time $t^{\prime}$. Denoting the magnetic field components by $G_{x}$ and $G_{y}$, respectively, we obtain

$$
\begin{aligned}
& G_{x}\left(x, y, z, t ; x^{\prime}, y^{\prime}, z^{\prime}, t^{\prime}\right)=-\frac{2 c\left(y-y^{\prime}\right) \tau}{\bar{r}^{2} \sqrt{c^{2} \tau^{2}-\bar{r}^{2}}} \delta\left(\sqrt{c^{2} \tau^{2}-\bar{r}^{2}}\right) \\
& G_{y}\left(x, y, z, t ; x^{\prime}, y^{\prime}, z^{\prime}, t^{\prime}\right)=\frac{2 c\left(x-x^{\prime}\right) \tau}{\bar{r}^{2} \sqrt{c^{2} \tau^{2}-\bar{r}^{2}}} \delta\left(\sqrt{c^{2} \tau^{2}-\bar{r}^{2}}\right)
\end{aligned}
$$

where $\tau=t-t^{\prime}$ and $\bar{r}=\sqrt{\left(x-x^{\prime}\right)^{2}+\left(y-y^{\prime}\right)^{2}}$.

\section{FIELDS INDUCED BY RELATIVISTIC BUNCH}

Let us assume the beam distribution function

$$
f(x, y, z)=\frac{1}{(2 \pi)^{3 / 2} \sigma_{x} \sigma_{y} \sigma_{z}} \exp \left(-\frac{x^{2}}{2 \sigma_{x}^{2}}-\frac{y^{2}}{2 \sigma_{y}^{2}}-\frac{z^{2}}{2 \sigma_{z}^{2}}\right) .
$$

The charge impinging elementary surface element $d x^{\prime} d y^{\prime}$ during time $d t^{\prime}$ is

$$
d q=c Q f\left(x^{\prime}, y^{\prime}, c t^{\prime}\right) d x^{\prime} d y^{\prime} d t^{\prime}
$$

where $Q$ is the total charge of the bunch. The magnetic field induced by the bunch is now obtained multiplying $d q$ by the Green function and integrating over $x^{\prime}, y^{\prime}$ and $t^{\prime}$. Let us first consider the $x$-component of the field $H_{x}$

$$
H_{x}(x, y, t)=c Q \int d x^{\prime} d y^{\prime} d t^{\prime} f\left(x^{\prime}, y^{\prime}, c t^{\prime}\right) G_{x}\left(x, y, z, t ; x^{\prime}, y^{\prime}, z^{\prime}, t^{\prime}\right) .
$$


Integration over $t^{\prime}$ can be done explicitly using the delta function in (2)

$$
\begin{aligned}
H_{x}(x, y, t) & =-2 Q c^{2} \int_{-\infty}^{\infty} d x^{\prime} d y^{\prime} \int_{-\infty}^{t} d t^{\prime} f\left(x^{\prime}, y^{\prime}, c t^{\prime}\right) \frac{\left(y-y^{\prime}\right) \tau}{\bar{r}^{2} \sqrt{c^{2} \tau^{2}-\bar{r}^{2}}} \delta\left(\sqrt{c^{2} \tau^{2}-\bar{r}^{2}}\right) \\
& =-2 Q \int d x^{\prime} d y^{\prime} f\left(x^{\prime}, y^{\prime}, c t-\bar{r}\right) \frac{y-y^{\prime}}{\bar{r}^{2}} .
\end{aligned}
$$

The last integral can be cast into dimensionless form

$$
\begin{aligned}
H_{x}(X, Y, T) & =-\frac{2 Q}{(2 \pi)^{3 / 2} \sigma_{x} \sigma_{y}} \int d X^{\prime} d Y^{\prime} \frac{Y-Y^{\prime}}{\rho^{2}} \\
& \exp \left(-\frac{X^{\prime 2}}{2 s_{x}^{2}}-\frac{Y^{\prime 2}}{2 s_{y}^{2}}-\frac{(T-\rho)^{2}}{2}\right),
\end{aligned}
$$

where we introduced the dimensionless variables

$$
T=\frac{c t}{\sigma_{z}}, X=\frac{x}{\sigma_{z}}, Y=\frac{y}{\sigma_{z}}, \rho=\frac{\bar{r}}{\sigma_{z}}, s_{x}=\frac{\sigma_{x}}{\sigma_{z}}, s_{y}=\frac{\sigma_{y}}{\sigma_{z}},
$$

and $\rho=\sqrt{\left(X-X^{\prime}\right)^{2}+\left(Y-Y^{\prime}\right)^{2}}$. To eliminate the singularity in the integrand of (7) it is convenient to use the polar coordinate system with the center at the observation point

$$
X^{\prime}-X=\rho \cos \phi, Y^{\prime}-Y=\rho \sin \phi .
$$

This gives

$$
\begin{aligned}
H_{x}(X, Y, T) & =-\frac{2 Q}{(2 \pi)^{3 / 2} \sigma_{x} \sigma_{y}} \int d \rho d \phi \sin \phi \\
& \times \exp \left(-\frac{(X+\rho \cos \phi)^{2}}{2 s_{x}^{2}}-\frac{(Y+\rho \sin \phi)^{2}}{2 s_{y}^{2}}-\frac{(T-\rho)^{2}}{2}\right) .
\end{aligned}
$$

To calculate the energy deposition we need to know the Fourier transform of the field (here and in what follows we use hat to denote Fourier transformed variables)

$$
\hat{H}_{x}=\frac{1}{2 \pi} \int_{-\infty}^{\infty} d t H_{x} e^{i \omega t}
$$


This can be computed analytically from (10)

$$
\begin{aligned}
\hat{H}_{x} & =-\frac{1}{2 \pi} \frac{2 Q \sigma_{z}}{(2 \pi)^{3 / 2} c \sigma_{x} \sigma_{y}} \int_{-\infty}^{\infty} d T e^{i \Omega T} \int d \rho d \phi \sin \phi \\
& \times \exp \left(-\frac{(X+\rho \cos \phi)^{2}}{2 s_{x}^{2}}-\frac{(Y+\rho \sin \phi)^{2}}{2 s_{y}^{2}}-\frac{(T-\rho)^{2}}{2}\right) \\
& =-\frac{2 Q \sigma_{z}}{(2 \pi)^{2} c \sigma_{x} \sigma_{y}} \int d \rho d \phi \sin \phi \\
& \times \exp \left(-\frac{(X+\rho \cos \phi)^{2}}{2 s_{x}^{2}}-\frac{(Y+\rho \sin \phi)^{2}}{2 s_{y}^{2}}-\frac{\Omega^{2}}{2}+i \Omega \rho\right),
\end{aligned}
$$

where $\Omega=\omega \sigma_{z} / c$.

Similarly to (12) one can obtain the Fourier transform of the $y$-component of the magnetic field

$$
\begin{aligned}
\hat{H}_{y} & =\frac{2 Q \sigma_{z}}{(2 \pi)^{2} c \sigma_{x} \sigma_{y}} \int d \rho d \phi \cos \phi \\
& \times \exp \left(-\frac{(X+\rho \cos \phi)^{2}}{2 s_{x}^{2}}-\frac{(Y+\rho \sin \phi)^{2}}{2 s_{y}^{2}}-\frac{\Omega^{2}}{2}+i \Omega \rho\right),
\end{aligned}
$$

\section{HEATING OF METAL BY IMAGE CURRENTS}

Penetration of the magnetic field inside the metal is described by the skin

effect, and with the assumption that the skin depth is smaller than the metal thickness is given by

$$
\hat{h}_{x}(z)=\hat{H}_{x} e^{i k z}, \quad \hat{h}_{y}(z)=\hat{H}_{y} e^{i k z}
$$

where $\hat{h}_{x}(z)$ and $\hat{h}_{y}(z)$ denote Fourier components of the magnetic field inside the metal, with

$$
k=(1+i) \sqrt{\frac{\sigma|\omega|}{2}},
$$

where $\sigma$ is the metal conductivity. The electric field in the metal is

$$
\hat{E}_{y}=\frac{i k}{\sigma} \hat{h}_{x}=(i-1) \sqrt{\frac{|\omega|}{2 \sigma}} \hat{H}_{x} e^{i k z}, \quad \hat{E}_{x}=-\frac{i k}{\sigma} \hat{h}_{y}=-(i-1) \sqrt{\frac{|\omega|}{2 \sigma}} \hat{H}_{y} e^{i k z}
$$


We will now calculate the total energy deposited due to the ohmic losses in the layer located immediately below the metal surface, at $z=0$; clearly this layer experiences the most intense heating. The total energy deposition over the time of passage of the bunch is given by the integral

$$
P=\sigma \int_{-\infty}^{\infty} d t\left[E_{x}^{2}(t)+E_{y}^{2}(t)\right]
$$

Expressing $E_{x}(t)$ and $E_{y}(t)$ through their inverse Fourier transforms

$$
E_{x}(t)=\int_{-\infty}^{\infty} d \omega \hat{E}_{x} e^{i \omega t}, \quad E_{y}(t)=\int_{-\infty}^{\infty} d \omega \hat{E}_{y} e^{i \omega t}
$$

and substituting into (17) we obtain

$$
\begin{aligned}
P & =\sigma \int_{-\infty}^{\infty} d t \int_{-\infty}^{\infty} d \omega d \omega^{\prime}\left[\hat{E}_{x}(\omega) \hat{E}_{x}\left(\omega^{\prime}\right)+\hat{E}_{y}(\omega) \hat{E}_{y}\left(\omega^{\prime}\right)\right] e^{i\left(\omega+\omega^{\prime}\right) t} \\
& =2 \pi \sigma \int_{-\infty}^{\infty} d \omega d \omega^{\prime}\left[\hat{E}_{x}(\omega) \hat{E}_{x}\left(\omega^{\prime}\right)+\hat{E}_{y}(\omega) \hat{E}_{y}\left(\omega^{\prime}\right)\right] \delta\left(\omega+\omega^{\prime}\right) \\
& =\pi \sigma \int_{0}^{\infty} d \omega\left[\left|\hat{E}_{x}(\omega)\right|^{2}+\left|\hat{E}_{y}(\omega)\right|^{2}\right]=\pi \int_{0}^{\infty} d \omega\left[\left|\hat{H}_{x}\right|^{2}+\left|\hat{H}_{y}\right|^{2}\right] \omega .
\end{aligned}
$$

Remarkably, the $\sigma$ is canceled in the last expression and $P$ does not depend on the conductivity of the metal.

Writing (12) and (13) as

$$
\hat{H}_{x}=\frac{Q \sigma_{z}}{2 \pi^{2} c \sigma_{x} \sigma_{y}} R_{x}\left(X, Y, \Omega, s_{x}, s_{y}\right), \quad \hat{H}_{y}=\frac{Q \sigma_{z}}{2 \pi^{2} c \sigma_{x} \sigma_{y}} R_{y}\left(X, Y, \Omega, s_{x}, s_{y}\right)
$$

with

$$
\begin{aligned}
R_{x}\left(X, Y, \Omega, s_{x}, s_{y}\right) & =-\int d \rho d \phi \sin \phi \\
& \times \exp \left(-\frac{(X+\rho \cos \phi)^{2}}{2 s_{x}^{2}}-\frac{(Y+\rho \sin \phi)^{2}}{2 s_{y}^{2}}-\frac{\Omega^{2}}{2}+i \Omega \rho\right) \\
R_{y}\left(X, Y, \Omega, s_{x}, s_{y}\right) & =\int d \rho d \phi \cos \phi \\
& \times \exp \left(-\frac{(X+\rho \cos \phi)^{2}}{2 s_{x}^{2}}-\frac{(Y+\rho \sin \phi)^{2}}{2 s_{y}^{2}}-\frac{\Omega^{2}}{2}+i \Omega \rho\right),
\end{aligned}
$$


we obtain for $P$

$$
P=\frac{Q^{2}}{4 \pi^{3} \sigma_{x}^{2} \sigma_{y}^{2}} H\left(X, Y, \Omega, s_{x}, s_{y}\right)
$$

where

$$
H\left(X, Y, \Omega, s_{x}, s_{y}\right)=\int_{0}^{\infty} \Omega d \Omega\left[\left|R_{x}\left(X, Y, \Omega, s_{x}, s_{y}\right)\right|^{2}+\left|R_{y}\left(X, Y, \Omega, s_{x}, s_{y}\right)\right|^{2}\right] .
$$

The expressions for the functions $R_{x}$ and $R_{y}$ and $H$ are simplified for the case of a round beam, $s_{x}=s_{y} \equiv s$. Due to the symmetry in this case it is clear that $H$ depends on the distance from the axis $R=\sqrt{X^{2}+Y^{2}}$ and can be computed as

$$
H(R, \Omega, s)=\int_{0}^{\infty} \Omega d \Omega\left|R_{x}(0, R, \Omega, s, s)\right|^{2}
$$

where, in turn,

$$
\begin{aligned}
& R_{x}(0, R, \Omega, s, s)=-\int d \rho d \phi \sin \phi \\
& \times \exp \left(-\frac{(\rho \cos \phi)^{2}}{2 s^{2}}-\frac{(R+\rho \sin \phi)^{2}}{2 s^{2}}-\frac{\Omega^{2}}{2}+i \Omega \rho\right) \\
& =-\int d \rho d \phi \sin \phi \exp \left(-\frac{R^{2}+\rho^{2}}{2 s^{2}}-\frac{R \rho \sin \phi}{s^{2}}-\frac{\Omega^{2}}{2}+i \Omega \rho\right) \\
& =2 \pi \exp \left(-\frac{R^{2}}{2 s^{2}}-\frac{\Omega^{2}}{2}\right) \int_{0}^{\infty} d \rho \exp \left(-\frac{\rho^{2}}{2 s^{2}}+i \Omega \rho\right) I_{1}\left(\frac{R \rho}{s^{2}}\right),
\end{aligned}
$$

with $I_{1}$ the modified Bessel function of the first order. One more integration can be carried out if (24) is written as

$$
\begin{aligned}
& H(R, \Omega, s)=4 \pi^{2} \exp \left(-\frac{R^{2}}{s^{2}}\right) \int_{0}^{\infty} \Omega d \Omega e^{-\Omega^{2}} \\
& \times \int_{0}^{\infty} d \rho \exp \left(-\frac{\rho^{2}}{2 s^{2}}+i \Omega \rho\right) I_{1}\left(\frac{R \rho}{s^{2}}\right) \int_{0}^{\infty} d \rho^{\prime} \exp \left(-\frac{\rho^{2}}{2 s^{2}}-i \Omega \rho^{\prime}\right) I_{1}\left(\frac{R \rho^{\prime}}{s^{2}}\right) .
\end{aligned}
$$




\section{PRACTICAL EXAMPLES}

Maximizing the function (23) over $X$ and $Y$ (or, in the case of a round beam maximizing the function (24) over $R$ ) and using the table properties of various metals, one can find for what charge of the beam $Q$ the foil will start to melt. In what follows we consider three metals: titanium, beryllium and tungsten. The relevant physical properties of the metals are presented in Table I.

TABLE I. Physical properties of titanium, Ti-6Al-4V alloy ${ }^{\mathrm{b}}$, beryllium, tungsten, and Ta-W alloy ${ }^{\mathrm{b}}$.

\begin{tabular}{|l|c|c|c|c|c|}
\hline Metal & Titanium & Ti-6Al-4V & Beryllium & Tungsten & Ta-W \\
\hline \hline Density $\left[\mathrm{g} / \mathrm{cm}^{3}\right]$ & 4.51 & 4.43 & 1.85 & 19.25 & 17.72 \\
\hline Melting point $\left[{ }^{\circ} \mathrm{C}\right]$ & 1668 & 1800 & 1287 & 3422 & 3000 \\
\hline Specific heat $[\mathrm{J} /(\mathrm{kg} \cdot \mathrm{K})]$ & 520 & 546 & 1820 & 132 & 131 \\
\hline Skin depth $[\mathrm{nm}]$ & 560 & 440 & 370 & 300 & $?$ \\
\hline$P_{\text {crit }}\left[\mathrm{kJ} /\left(\mathrm{cm}^{3}\right)\right]$ & 3.9 & 4.3 & 4.3 & 8.6 & 6.9 \\
\hline
\end{tabular}

a Ti-6Al-4V contains $90 \% \mathrm{Ti}, 6 \% \mathrm{Al}$ and $4 \% \mathrm{~V}$.

b Ta-W contains $58.5 \%$ Ta and $41.5 \% \mathrm{~W}$.

The skin depth in the table is calculated for the frequency corresponding to the bunch length of $20 \mu \mathrm{m}\left(\omega=c / \sigma_{z}\right)$ using the metal resistivity at the melting temperature for titanium, beryllium and tungsten (this resistivity is 7.3, 32.7 and 17.3 times larger than the nominal ones $\left[2.5 \times 10^{6} 1 /(\mathrm{Ohm} \cdot \mathrm{m})\right.$, $2.5 \times 10^{7} 1 /(\mathrm{Ohm} \cdot \mathrm{m})$ and $2.0 \times 10^{7} 1 /(\mathrm{Ohm} \cdot \mathrm{m})$, respectively] at $20^{\circ} \mathrm{C}$. For the alloy Ti-6Al-4V we used the conductivity of $0.54 \times 10^{6} 1 /(\mathrm{Ohm} \cdot \mathrm{m})$ at temperature of $560^{\circ} \mathrm{C}$.

The last line in the table shows the critical energy density $P_{\text {crit }}$ needed to 
heat the metal from $20^{\circ} \mathrm{C}$ to the melting point. This quantity is used below to calculate the beam charge which melts the surface of the foil when it passes through it due to the dissipation of the image currents. Note that the value of $P_{\text {crit }}$ is the same for Ti- $6 \mathrm{Al}-4 \mathrm{~V}$ and Beryllium, which means that critical bunch charges are the same for the both metals.

As a first example we take $\sigma_{x}=\sigma_{y}=20 \mu \mathrm{m}$ and consider a bunch length $\sigma_{z}$ variable from the minimal value $\sigma_{z}=10 \mu \mathrm{m}$ to the maximal value of 100 $\mu \mathrm{m}$; this corresponds to the parameter $s$ in (24) varying from 2 to 0.2 . The plot of $\max H$ in this case is shown in Fig. 1. The plot of the critical bunch

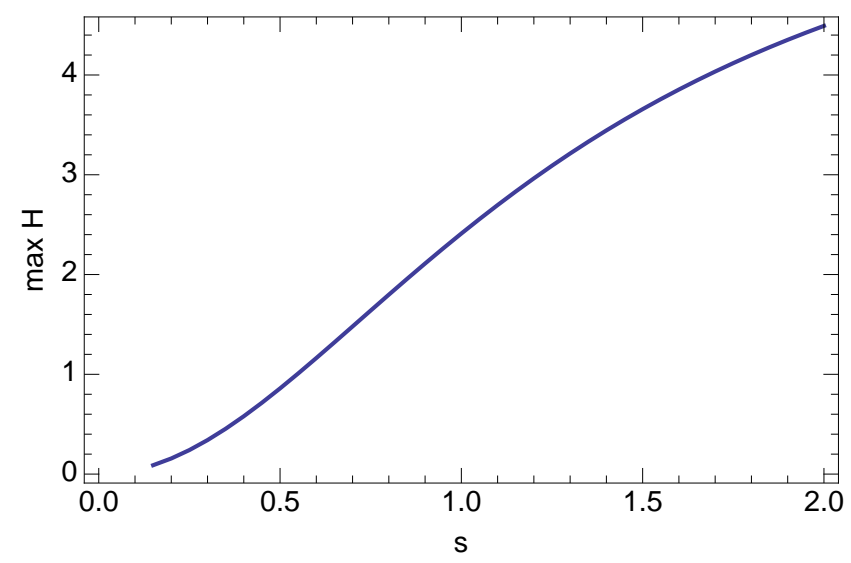

FIG. 1. Plot of $\max H$ versus $s$.

charge at which the melting begins for a given metal is shown in Fig. 2 as a function of the bunch length. The critical bunch charge for a round beam with all dimensions of $20 \mu \mathrm{m}$ is shown in Table II.

We also found the maximum value of $H$ for the case $s_{x}=1$ and $s_{y}=5$, which is equal to 5.4. This value can be used to evaluate the melting condition for the beam with $\sigma_{x}=\sigma_{z}=20 \mu \mathrm{m}$ and $\sigma_{y}=100 \mu \mathrm{m}$. The result is shown in Table III. 


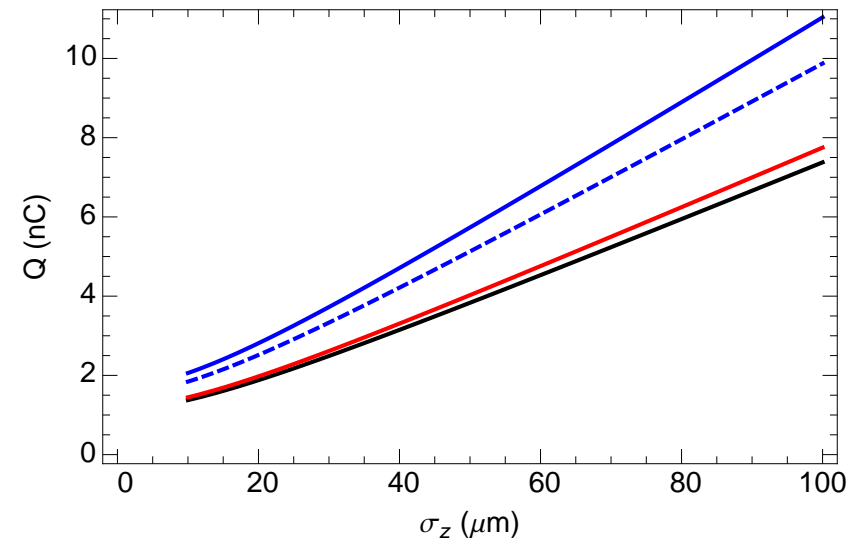

FIG. 2. Critical bunch charge for titanium (black), berrylium and Ti-6Al-4V (red), Ta-W (dashed blue) and tungsten (blue) as a function of bunch length (assuming $\left.\sigma_{x}=\sigma_{y}=20 \mu \mathrm{m}\right)$.

TABLE II. Critical bunch charge for $\sigma_{x}=\sigma_{y}=\sigma_{z}=20 \mu \mathrm{m}$.

\begin{tabular}{|c|c|c|c|c|c|}
\hline Metal & Titanium & Ti-6Al-4V & Beryllium & Ta-W & Tungsten \\
\hline \hline Q [nC] & 1.9 & 2.0 & 2.0 & 2.5 & 2.8 \\
\hline
\end{tabular}

\section{ACKNOWLEDGEMENTS}

The author thanks V. Yakimenko, who raised the question of melting foils with impinging relativistic beams, and M.Zolotorev, for useful discussions. The author is thankful to D. Walz for providing data for Ti-6Al-4V and Ta-W 
TABLE III. Critical bunch charge for $\sigma_{x}=\sigma_{z}=20 \mu \mathrm{m}$ and $\sigma_{y}=100 \mu \mathrm{m}$.

\begin{tabular}{|c|c|c|c|c|c|}
\hline Metal & Titanium & Ti-6Al-4V & Beryllium & Ta-W & Tungsten \\
\hline \hline Q [nC] & 6.3 & 6.6 & 6.6 & 8.4 & 9.4 \\
\hline
\end{tabular}

alloys.

[1] A. W. Chao, Physics of Collective Beam Instabilities in High Energy Accelerators (Wiley, New York, 1993). 